\title{
HÁBITOS DEL SUEÑO Y ACCIDENTES DE TRÁNSITO EN CONDUCTORES DE ÓMNIBUS INTERPROVINCIAL DE AREQUIPA, PERÚ
}

\author{
Alex Caso ${ }^{1, a}$, Jorge Rey de Castro 2,3,4,b, Edmundo Rosales-Mayor ${ }^{4,5, c}$
}

\begin{abstract}
RESUMEN
Con el objetivo de determinar los hábitos de sueño en los conductores de ómnibus y su relación con los accidentes en la ciudad de Arequipa, Perú, se realizó un estudio transversal y descriptivo en una muestra no probabilística de 166 conductores. Las horas de conducción por día fueron de 9,4 $\pm 3,7$; el 54\% (89) conduce más de 4 h sin detenerse; 74\% (123) conducen de noche y $87 \%$ (145) duermen en el ómnibus. El 75\% reconoció cansancio durante la conducción (124). El 27\% (45) tuvo somnolencia; el 24\% (40) refirió haberse accidentado o haber estado a punto de accidentarse durante la conducción. La somnolencia o el cansancio durante la conducción fue frecuente en esta población, y sus hábitos de conducción y descanso podrían propiciarlo.
\end{abstract}

Palabras clave: Accidentes de tránsito; Somnolencia; Fatiga; Privación del sueño (fuente: DeCS BIREME).

\section{SLEEP HABITS AND TRAFFIC ACCIDENTS IN INTER-PROVINCIAL BUS DRIVERS OF AREQUIPA, PERU}

\begin{abstract}
In order to determine sleep habits in bus drivers and their relationship to accidents in the city of Arequipa, Peru, a crosssectional descriptive study was conducted in a non-probabilistic sample of 166 drivers. Driving hours per day were 9.4 \pm 3.7 . 54\% (89) drive over 4 hours without stopping; 74\% (123) drive at night; and $87 \%$ (145) sleep on the bus. $75 \%$ reported fatigue while driving (124). 27\% (45) had drowsiness; $24 \%$ (40) reported having been in or on the verge of an accident while driving. Sleepiness or fatigue while driving was common in this population and their driving and rest habits could contribute to this.
\end{abstract}

Key words: Accidents, traffic; Sleep stages; Fatigue; Sleep deprivation (source: MeSH, NLM).

\section{INTRODUCCIÓN}

Los accidentes de tránsito constituyen una problemática mundial y causan una mortalidad y morbilidad anual de 1,24 millones y 20-50 millones de personas respectivamente (1). En el Perú, el año 2013, el Ministerio de Transportes y Comunicaciones (MTC) reportó 59453 heridos y 3110 fallecidos ${ }^{(2)}$. Diversos estudios revelan que la somnolencia durante la conducción es causante de accidentes y que la siniestralidad puede disminuir si se toman medidas correctivas ${ }^{(3)}$. La National Transportation Safety Board de EE. UU. menciona que la somnolencia es responsable del
$52 \%$ de colisiones en camiones de carga y $30 \%$ de los accidentes mortales de vehículos comerciales pesados ${ }^{(4)}$. En Europa ocasiona el $20 \%$ de accidentes de vehículos de transporte comercial ${ }^{(5)}$. En Perú, en las estadísticas del año 2013 del MTC (6), se considera al exceso de velocidad como la principal causa de accidentes (32\%) seguida por imprudencia (28\%) y ebriedad (12\%) del conductor. Estas fuentes oficiales no registran a la somnolencia como causa de accidentes, sin embargo, disponemos de información relacionada al tema que documenta lo contrario ${ }^{(7-13)}$. La ciudad de Arequipa fue la tercera ciudad con mayor flujo de pasajeros el $2010^{\text {(14). }}$

\footnotetext{
1 Universidad Católica de Santa María. Arequipa, Perú.

2 CENTRES Clínica Anglo Americana. Lima, Perú.

3 Universidad Peruana Cayetano Heredia. Lima, Perú.

4 Grupo de Investigación en Sueño. Lima, Perú.

5 Servicio de Neumología, Instituto Clínic del Tórax, Hospital Clínic de Barcelona. Barcelona, España.

a Médico Cirujano

b Médico neumólogo y trastornos respiratorios del sueño. Magíster en Medicina.

c Médico neumólogo. Magíster en Medicina. Máster en sueño: fisiología y medicina.

Recibido: 05-04-14 Aprobado: 20-08-14
}

Citar como: Caso A, Rey de Castro J, Rosales-Mayor E. Hábitos del sueño y accidentes de tránsito en conductores de ómnibus interprovincial de Arequipa, Perú. Rev Peru Med Exp Salud Publica. 2014;31(4):707-11. 
Tabla 1. Características de las rutas según destino

\begin{tabular}{lcccc}
\hline Características & Ruta 1* & Ruta 2 & Ruta 3 & Ruta 4 \\
\hline Destinos & Cerro verde & $\begin{array}{c}\text { Provincias dentro de } \\
\text { Arequipa }\end{array}$ & $\begin{array}{c}\text { Departamentos aledaños a } \\
\text { Arequipa }\end{array}$ & Destino Lima \\
Distancias & $32 \mathrm{~km}$ & 40 a 178 km & 160 a $515 \mathrm{~km}$ & $1000 \mathrm{~km}$ \\
$\begin{array}{l}\text { Conductores por } \\
\text { recorrido }\end{array}$ & 1 & 1 & 1 y/0 2 & 2 \\
Carretera & Asfaltada & Trocha & Asfaltadas & Asfaltadas \\
Horarios de salida & $20: 00-22: 00$ y 03:00-05:00 h & $4: 00-22: 00 \mathrm{~h}$ & $4: 00-22: 30 \mathrm{~h}$ & $16: 00-20: 00 \mathrm{~h}$ \\
Encuestas & $26(16 \%)$ & $63(38 \%)$ & $32(19 \%)$ & $44(27 \%)$ \\
\hline
\end{tabular}

* Dos sistemas de rotación: 1) alternan 15 días de trabajo diurno con 15 días de trabajo nocturno (15x15) sin descanso; y 2) alternan 7 días de trabajo diurno con 7 días de trabajo nocturno (7x7) sin descanso

El presente estudio tiene como objetivo indagar los hábitos de conducción y de sueño en los conductores de ómnibus interprovincial de la ciudad de Arequipa y explorar su relación con los accidentes de tránsito.

\section{EL ESTUDIO}

Estudio de diseño transversal y descriptivo, con muestreo no probabilístico y por conveniencia, de los conductores de ómnibus de la ciudad de Arequipa, Perú. La población estuvo formada por conductores de las empresas que ofrecen sus servicios en el Terminal Terrestre Corattsa y en el Terrapuerto. Las empresas cubren rutas dentro del departamento de Arequipa hacia departamentos aledaños y localidades más distantes como la ciudad de Lima. Los diferentes destinos fueron usados para una clasificación arbitraria de grupos de conductores en cuatro rutas. La ruta 1 utiliza dos sistemas de rotación (Tabla 1).

Unas 58 empresas operan en ambas terminales, de ellas se seleccionaron dieciséis al azar, nueve aceptaron participar. No hay registro formal de conductores, sin embargo, se estimó un aproximado de 470 conductores; participaron 197 y se excluyeron 31 por tener cuestionarios incompletos. Se analizaron 166 cuestionarios. El estudio fue implementado en 5 días consecutivos en diciembre de 2010. Los conductores de la ruta 1 se encontraban en el período de rotación nocturna.

Se utilizó un cuestionario validado y empleado en estudios previos en nuestro medio (7-11) que evalúa hábitos de conducción y de sueño. Se utilizó además la escala de somnolencia de Epworth (ESE) ${ }^{(15)}$, se consideró presencia de excesiva somnolencia diurna (ESD) un puntaje mayor a 10. La entrevista fue voluntaria y anónima y el tiempo promedio fue 10 minutos. Se realizó una prueba piloto con 15 conductores. Definimos bodega o maletero como el lugar empleado para guardar las maletas y que se encuentra ubicado en la parte inferior del ómnibus. Cabina de descanso es un cubículo ubicado en la parte posterior del asiento del conductor. Los conductores suelen emplear los términos cansancio y somnolencia como sinónimos ${ }^{(10)}$.

Se presenta la estadística descriptiva adecuada a la naturaleza de las variables. Para la descripción de las variables cuantitativas se utilizarán medidas de tendencia central (media, mediana) y medidas de dispersión (desviación estándar). Para las variables cualitativas, se utilizarán frecuencias y porcentajes (por defecto sobre el total de respuestas) de cada una de las respuestas posibles. El estudio fue revisado y aprobado por un Comité Institucional de Ética de la Investigación. Todos los participantes firmaron un consentimiento informado antes de la entrevista.

\section{RESULTADOS}

Todos los conductores fueron varones con una edad promedio 44,2 $\pm 8,7$ años. El 29\% no tenía seguro médico contra accidentes de tránsito y el 33\% no recibía pago por vacaciones $33 \%$. La sistemática de trabajo y descanso se describen en la Tabla 2. Conducen durante la noche 123 (74\%). El 54\% de los conductores de las rutas 2, 3 y 4 conducen de noche, y lo hacen entre 5 a 7 noches por semana. Veintiún (81\%) conductores de la ruta 1 trabajan

Tabla 2. Características generales de los conductores, según ruta

\begin{tabular}{lccccc}
\hline & Ruta 1 & Ruta 2 & Ruta 3 & Ruta 4 & Total \\
\hline $\begin{array}{l}\text { Horas de conduc- } \\
\text { ción en 24 h }\end{array}$ & $8,3 \pm 2,3$ & $8,5 \pm 3,8$ & $9,8 \pm 3,6$ & $10,9 \pm 3,1$ & $9,4 \pm 3,7$ \\
$\begin{array}{l}\text { Conducción } \geq 4 \mathrm{~h} \\
\text { sin detenerse }\end{array}$ & $5(17 \%)$ & $24(38 \%)$ & $26(81 \%)$ & $34(77 \%)$ & $89(54 \%)$ \\
$\begin{array}{l}\text { Conducción } \\
\text { nocturna }\end{array}$ & $27(100 \%)$ & $35(56 \%)$ & $25(78 \%)$ & $36(82 \%)$ & $123(74 \%)$ \\
$\begin{array}{l}\text { Horas de sueño } \\
\text { en 24 h }\end{array}$ & $5,8 \pm 1,5$ & $7,1 \pm 1,7$ & $6,1 \pm 1,9$ & $7,5 \pm 1,5$ & $6,6 \pm 1,7$ \\
$\begin{array}{l}\text { Horas de sueño el } \\
\text { día de descanso }\end{array}$ & $7,7 \pm 1,8$ & $8,5 \pm 1,5$ & $7,8 \pm 2,1$ & $9,0 \pm 1,6$ & $8,25 \pm 1,8$ \\
$\begin{array}{l}\text { Durmió en las } \\
\text { últimas 24 h }\end{array}$ & $5,6 \pm 1,6$ & $7,4 \pm 1,4$ & $6,9 \pm 2,1$ & $7,6 \pm 1,5$ & $6,9 \pm 1,7$ \\
$\begin{array}{l}\text { Accidentarse o ha- } \\
\text { ber estado a punto } \\
\text { de accidentarse }\end{array}$ & $6(22 \%)$ & $13(21 \%)$ & $14(44 \%)$ & $7(16 \%)$ & $40(24 \%)$ \\
\hline
\end{tabular}




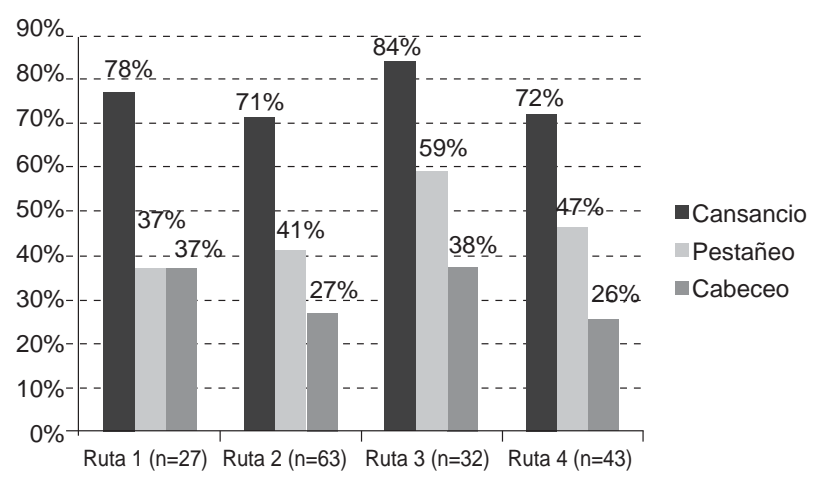

Figura 1. Cansancio, pestañeo y cabeceo durante la conducción por rutas

con sistema en el que alternan 15 días de tranajo diurno y 15 días de trabajo nocturno sin descanso y los otros 5 conductores (19\%) con sistema que alternan 7 días de trabajo diurno y 7 días de trabajo nocturno sin descanso.

Ciento veinticuatro $(75 \%)$ reconocieron cansancio, $75(45 \%)$ pestañeo y $40(30 \%)$ cabeceo durante la conducción (Figura 1). En relación al momento del día en que el conductor tuvo más cansancio, 41 (34\%) refirieron sentirlo durante las madrugadas, 37 (31\%) en las noches y $34(29 \%)$ durante las tardes. La maniobra empleada con más frecuencia para controlar el cansancio durante la conducción fue escuchar música (Figura 2).

Ciento cuarenta y cinco (87\%) conductores dormían en el ómnibus cuando este se encontraba en la terminal y/o durante su recorrido. El lugar del ómnibus más usado para dormir fue la bodega (32\%), seguido por un asiento de pasajero (24\%) y luego la cabina de descanso $(13 \%)$, el $31 \%$ restante alternaba entre la bodega y un asiento de pasajero. Refirieron ronquidos, pausas respiratorias

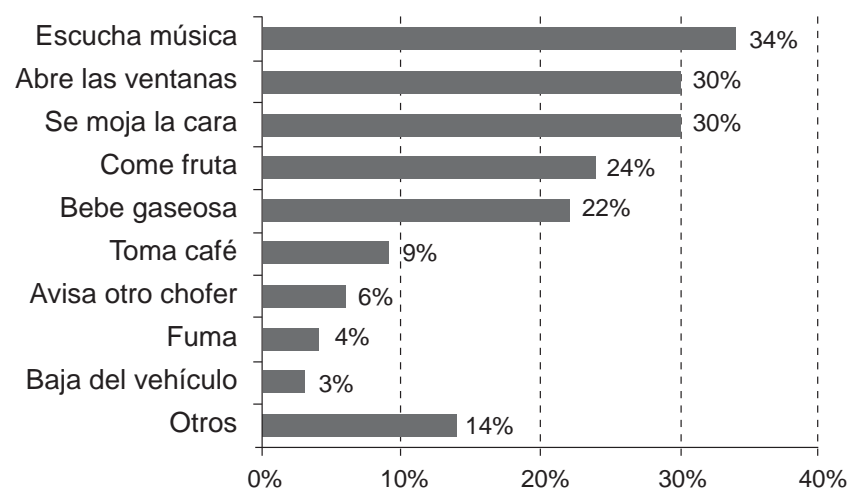

Figura 2. Medidas implementadas para no dormirse durante la conducción $(n=155)$ y somnolencia diurna 70,28 y $27 \%$ respectivamente. De acuerdo con la ESE, $27 \%$ presentaron ESD.

Cuarenta (24\%) conductores refirieron haberse accidentado o haber estado a punto de accidentarse (Tabla 2). Por un lado, los conductores manifestaron que la principal causa fue el cansancio (54\%), seguido por imprudencia del otro chofer $(26 \%)$, mal estado de las carreteras $(8 \%)$, falla mecánica $(5 \%)$, presencia de neblina $(2 \%)$, y otros $(5 \%)$. Por otro lado, $44 \%$ de los encuestados sabían de otros conductores que tuvieron accidentes debido al cansancio. De acuerdo a la opinión de los conductores, $77 \%$ opina que el cansancio es la principal causa de accidentes en las carreteras seguido por la imprudencia del conductor $(66 \%)$ y fallas mecánicas (30\%).

\section{DISCUSIÓN}

Este estudio confirma la casi inexistente prestación social básica para los conductores, como son el seguro de salud y las vacaciones pagadas. Un alto porcentaje conduce más de cuatro horas sin detenerse, incumpliendo las normativas y reglamentos existentes en nuestro medio ${ }^{(16)}$. Las encuestas aplicadas en los estudios de Fiori ${ }^{(7)}$ y Huancayo ${ }^{(8)}$ encontraron un 26 y $80 \%$, respectivamente. De acuerdo a este marco normativo la duración acumulada de jornadas de conducción no debe exceder las diez horas en un periodo de $24 \mathrm{~h}{ }^{(16)}$. En conductores de la ruta 4 esta pauta no se cumple. La norma obliga a emplear dos conductores a lo largo de todo el recorrido si el tiempo total de conducción es mayor de 5 o $4 \mathrm{~h}$ en el día o noche ${ }^{(16)}$. Dos conductores alternan el volante y mientras uno conduce el otro descansa en el interior del bus sin tener la oportunidad de un sueño reparador en una habitación confortable. Esta sistemática de alternancia al volante es promocionada en términos de mayor seguridad. Nuestros estudios demuestran que esta normativa aplicada cotidianamente en el país no garantiza al pasajero un viaje seguro ${ }^{(7-13)}$.

Tres de cada cuadro conductores conducían de noche y este hallazgo se repite en los estudios de Fiori (7), Huancayo ${ }^{(8)}$ y Lima ${ }^{(9)}$. Ciertamente, los pasajeros prefieren el turno nocturno con la intención de evitar gastos de hotelería y las empresas de transporte se adaptan a esa demanda. En las rutas 2, 3 y 4 sesenta y ocho por ciento trabaja cuatro o más noches por semana y en la ruta 1 emplea un sistema de rotación sin descanso de por medio. Los hallazgos confirman la gran carga laboral de los conductores, especialmente nocturna. No hemos encontrado precedentes relacionados a sistemas de vigilia-descanso en la literatura relacionada con este tema. 
La pérdida de sueño compromete la vigilancia, la memoria, el lenguaje, la motivación, el juicio y el desempeño cognitivo y académico ${ }^{(17,18)}$. Los hábitos de sueño identificados por medio de la encuesta denotan privación crónica del sueño. La habitual sintomatología está relacionada a somnolencia y/o cansancio, particularmente incrementados durante las madrugadas y tardes, de acuerdo al patrón normal del sueño y su comportamiento circadiano. Las tres cuartas partes tienen sensación de cansancio durante su actividad laboral y suelen emplear un sinnúmero de maniobras para controlarlo con la certeza que funcionan. Hallazgos similares han sido descritos en Fiori ${ }^{(7)}$, Huancayo ${ }^{(8)}$ y Lima ${ }^{(9)}$.

Se encuentra alta frecuencia de conductores que duermen en el ómnibus. Este hábito al parecer, esta enraizado en las empresas de transporte de pasajeros del Perú $(7,8)$. En algunos casos, duermen en el maletero del ómnibus cuando está en la terminal terrestre o durante el recorrido en ruta. Otros utilizan los asientos de los pasajeros o la cabina de descanso. No es posible lograr una recuperación del sueño en estas condiciones y mucho menos en un periodo de 4 o $5 \mathrm{~h}$ en el que alternan la conducción con otro conductor.

Las altas frecuencias de cabeceo y pestañeo reconocidos indican el estado de cansancio crónico en que laboran los conductores. Los hallazgos se repiten en otros estudios (7,8). Llamó la atención las frecuencias elevadas de los síntomas cardinales del síndrome de apneas-hipopneas del sueño (ronquidos, pausa respiratoria y somnolencia) aunque no es posible extraer mayores conclusiones ya que no se implementaron registros de monitoreo del sueño.

Se debe enfatizar la diferencia entre el puntaje de la ESE y la información relacionada a la sensación de cansancio referida por los mismos conductores. Ambas variables son subjetivas, difíciles de cuantificar y comparar. Utilizar un cuestionario universalmente conocido como la ESE nos permitió hacer comparaciones con otros estudios. En el grupo de conductores arequipeños se encontró un mayor puntaje en la escala de somnolencia y un mayor porcentaje de conductores con ESD en comparación con los conductores de la ciudad de Huancayo ${ }^{\left({ }^{8}\right)}$.

Está documentado que los accidentes relacionados con la somnolencia son diez veces más frecuentes en la noche que durante el día ${ }^{(19)}$ y es previsible que el riesgo se incremente si el conductor duerme pocas horas, tiene sueño de mala calidad o que trabaje casi exclusivamente de noche ${ }^{(20)}$; características muy prevalentes en la muestra estudiada.

Más de la cuarta parte de conductores admitieron haber sufrido un accidente y/o haber estado a punto de accidentarse. Estas cifras fueron menores a las halladas en Lima ${ }^{(7)}$ y Huancayo ${ }^{(8)}$ aunque no por ello dejan de ser alarmantes. Más de la mitad de encuestados indicaron que el cansancio fue la principal causa de dicho evento. Llama la atención la mayor frecuencia de dicho evento en los conductores de la ruta 3. Esto podría deberse a que es una de las rutas de mayor distancia aunque el número de encuestados por ruta no permite extraer mayores conclusiones. Cuando se solicitó a todos los encuestados indicar cuál era la principal causa de accidentes en el Perú, más de las tres cuartas partes mencionaron al cansancio.

El presente estudio tiene las limitaciones inherentes al empleo de cuestionarios, herramientas de carácter subjetivo, sesgo de memoria y problemas relacionados con la veracidad de la información brindada por el conductor debido a potenciales represalias laborales y/o penalidades por no cumplir con las normas. Dichos factores se intentaron controlar implementando una encuesta anónima y voluntaria. La segunda limitación está relacionada con la muestra no probabilística, ya que no se dispone de registros formales que permitan cuantificar el universo de conductores. A pesar de estas limitaciones se puede considerar que los resultados son relevantes en la perspectiva de la salud pública y la seguridad.

Aunque el diseño de este estudio no permite establecer causalidad, los resultados sugieren que en Arequipa la somnolencia y/o cansancio podrán ser causa de accidentes de tránsito en conductores de ómnibus de pasajeros. Este hallazgo es consistente con los estudios previos en este grupo ${ }^{(7-9,12)}$ es por ello que se puede sustentar que los accidentes de tránsito en el Perú debido a la somnolencia y/o cansancio del conductor son una realidad. Lamentablemente, el MTC no registra esta variable de causalidad en los accidentes y las instituciones regulatorias al no tenerlo identificado, no están en condiciones de implementar intervenciones para corregirlo. Es necesario señalar que el entorno informal en el que vive el sistema de transportes en el Perú implica un gran reto para las autoridades.

Es aconsejable implementar investigaciones similares dirigidas a otras poblaciones de conductores como microbuses, combis, taxistas y vehículos particulares con la finalidad de explorar estas variables como causas de accidentes en zonas urbanas. Las intervenciones generales para disminuir el riesgo de accidentes de ómnibus en las carreteras del país han sido planteadas anteriormente ${ }^{(13)}$ y urge implementarlas.

Contribuciones de autoría: AC, JRC y ER han participado en la concepción y diseño del artículo, su redacción y aprobación de la versión final y la asesoría técnica. Además AC y JRC 
realizaron la recolección y obtención de resultados, el análisis e interpretación de datos, y AC obtuvo el financiamiento y la asesoría estadística.
Fuentes de financiamiento: autofinanciada.

Conflictos de interés: ninguno.

\section{REFERENCIAS BIBLIOGRÁFICAS}

1. World Health Organization. Global status report on road safety 2013. Geneva: WHO; 2013.

2. Perú, Ministerio de Transportes y Comunicaciones. Número de víctimas de accidentes de tránsito fatales y no fatales por año, según características de las víctimas: 2004-2013 [Internet]. Lima: MTC; 2013 [citado el 01 de agosto de 2014]. Disponible en: http://www.mtc.gob.pe/estadisticas/ files/cuadros/Transportes_ Carretero_2_5_3.xlsx

3. Connor J, Norton R, Ameratunga S, Robinson E, Civil I, Dunn R, et al. Driver sleepiness and risk of serious injury to car occupants: population based case control study. Bmj. 2002;324(7346):1-5.

4. National Transportation Safety Board. Evaluation of U.S. Department of Transportation Efforts in the 1990s to Address Operator Fatigue. Safety Report NTSB/SR-99/01 [Internet]. Washington, D.C.: NTSB; 1999 [actualizado 1999; citado el 30 de octubre de 2013]. Disponible en: http://www.ntsb.gov/doclib/ reports/1999/SR9901.pdf

5. European Transport Safety Council. The role of driver fatigue in commercial road transport crashes. Bruselas: ETSC; 2001 [actualizado 2001; citado el 30 de octubre de 2013]. Disponible en: http://www.etsc.eu/documents/ drivfatigue.pdf

6. Perú, Ministerio de transportes y Comunicaciones. Número de accidentes de tránsito fatales y no fatales por año, según características de las victimas: 2004-2013 [Internet]. Lima: MTC; 2013 [citado el 1 de agosto del 2014]. Disponible en: http://www. mtc.gob.pe/estadisticas/files/cuadros/ Transportes_Carretero_2_5_3.xlsx

7. Rey de Castro J, Gallo J, Loureiro H. [Tiredness and sleepiness in bus drivers and road accidents in Peru: a quantitative study]. Rev Panam Salud Publica. 2004 Jul;16(1):11-8.
8. Rosales E, Egoavil M, Durand I, Montes N, Flores R, Rivera S, et al. Accidentes de carretera y su relación con cansancio y somnolencia en conductores de ómnibus. Rev Med Hered. 2009;20(2):48-59.

9. Liendo GR, Castro CL, Rey de Castro J. Cansancio y somnolencia en conductores de ómnibus interprovinciales: estudio comparativo entre formalidad e informalidad. Rev Peru Med Exp Salud Publica. 2010 Abr-Jun;27(2):187-94.

10. Rey de Castro J, Soriano S. Hipersomnia durante la conducción de vehículos ¿causa de accidentes en carreteras? A propósito de un estudio cualitativo. Rev Soc Peru Med Interna. 2002;15(3):142-9.

11. Rey de Castro J. Accidentes de tránsito en carreteras e hipersomnia durante la conducción. ¿Es frecuente en nuestro medio? La evidencia periodística. Rev Med Hered. 2003;14(2):69-73.

12. Deza-Becerra F, Gonzales-Gonzales C. Hábitos del sueño, cansancio y somnolencia diurna en conductores de transporte inter provincial de Chiclayo. 2012. IV Congreso de Federaciones Latinoamericanas de Sociedades del Sueño y X Congreso Colombiano de Medicina del Sueño. Chiclayo, Perú. 14 al 17 de noviembre del 2012.

13. Rey de Castro J, Rosales-Mayor E. Cansancio y somnolencia durante el desempeño laboral de los conductores interprovinciales: experiencia peruana y planteamiento de propuestas. Rev Peru Med Exp Salud Publica. 2010 Abr-Jun;27(2):237-42.

14. Perú, Ministerio de Transportes y Comunicaciones. Tráfico de pasajeros en el transporte interprovincial, según departamento destino: 2004-2013. Lima: MTC; 2009 [citado el 01 de agosto de.l 2014]. Disponible en: http://www.mtc.gob.pe/estadisticas/ files/cuadros/Transportes_ Carretero_2_1_1.xlsx
15. Rosales-Mayor E, Rey de Castro J, Huayanay L, Zagaceta K. Validation and modification of the Epworth Sleepiness Scale in Peruvian population. Sleep Breath. 2012 Mar;16(1):59-69. doi: 10.1007/s11325-011-0485-1.

16. Perú, Ministerio de Transportes y Comunicaciones. Decreto Supremo 040-2011-MTC: Decreto Supremo que modifica el Reglamento Nacional de Licencias de Conducir Vehículos Automotores y No Motorizados de Transporte Terrestre, aprobado por Decreto Supremo N ${ }^{\circ}$ 040-2008-MTC; el Reglamento Nacional de Transporte Público Especial de Pasajeros en Vehículos Motorizados o No Motorizados, aprobado por Decreto Supremo $\quad \mathrm{N}^{\circ}$ 055-2010-MTC e incorpora disposiciones al Reglamento Nacional de Administración de Transporte, aprobado por Decreto Supremo No 017-2009-MTC. El Peruano. 31 de diciembre del 2011.

17. Pilcher JJ, Huffcutt AI. Effects of sleep deprivation on performance: a metaanalysis. Sleep. 1996 May;19(4):31826.

18. Curcio G, Ferrara M, De Gennaro L. Sleep loss, learning capacity and academic performance. Sleep Med Rev 2006;10(5):323-37.

19. Peden MM, Scurfield R, Sleet D, Mohan D, Hyder A, Jarawan E, et al; World Health Organization. World report on road traffic injury prevention. Geneva: WHO; 2004.

20. Hamelin P. Lorry driver's time habits in work and their involvement in traffic accidents. Ergonomics. 1987 Sep;30(9):1323-33.

Correspondencia: Jorge Rey de Castro.

Dirección: Av. Alfredo Salazar 314-1004 San Isidro, Lima 27, Perú.

Teléfono: (511) 2219013

Correo electrónico:jreydecastrom@gmail.com 